\title{
Opportunistic XOR-Coding Content Propagation in VANET
}

\author{
Hossein Malakooti, Ali Jalooli, Mohammad Reza Jabbarpour, Alireza Marefat, and Rafidah Md Noor
}

\begin{abstract}
The main difference between typical wireless networks and Vehicular Ad-hoc Networks is mostly about the way traffic is propagated in these types of networks. Unlike conventional wireless networks in VANET connections are established quick and short. Because of these two factors connections in VANET are not as reliable and robust as typical wireless networks. Hence Due to these limitations in VANET and also in order to achieve higher throughput, we are aimed at investigating the adoption of network coding with the existing data propagation and routing methods in Vehicular networks. In order to address the disadvantages, we present an approach called Opportunistic Network Coding-aware Data Dissemination in Wireless Networks (NCODWN). This approach is an enhanced version of COAR. We have managed to improve network throughput and decrease end-to-end delay of the network by implementing a more efficient and reliable mechanism. The simulation results indicate that our approach outperforms COAR in terms of end-to-end delay, percentage of encoded packets, packet delivery and network throughput.
\end{abstract}

Index Terms-Data propagation, network coding, vehicular ad hoc network, wireless.

\section{INTRODUCTION}

The emergence of wireless networks has made a crucial impact on network communications. Over the last four decades of research were conducted on the different aspects of communication between mobile networks and especially vehicular networks. One of the most important challenges in VANET is considerable rates of packet loss and insufficient throughput. Network coding (NC) [1] was introduced with the main aim of increasing the network throughput. Fig. 1 represents basic concepts of NC. In a wider overview due to nature of wireless networks, they suffer from low throughput and do not scale well in heavy implementations[2] which this problem is inherited by VANET.

\section{A. Network Coding and Routing Protocols}

In order to utilize NC in wireless network in a practical way, one of the most well-known applications is in routing protocols. In other words, $\mathrm{NC}$ is applied in routing In order to create a coding-aware routing scheme. Existing NC-aware routing mechanisms in VANET evaluate links only based on possibility and impossibility of coding in nodes.

Being aware of Applicable NC scenarios and opportunistic coding among different flows at certain amount of time provides the opportunity to improve the throughput of networks. Thus in order to Improve performance of existing

Manuscript received January 9, 2013; revised March 27, 2014.

The authors are with the University of Malaya, Malaysia (email: hossein.malakooti@gmail.com, fidah@um.edu.my) protocols it is important to improve a NC-Aware routing to support active XOR coding between various data flows [3].

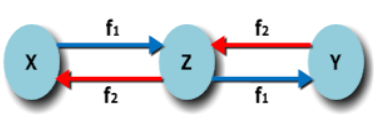

(a)

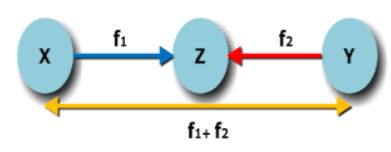

(b)
Fig. 1. Wireless network scenarios (a) network coding is not applied (b) network coding is applied.

\section{B. Opportunistic Routing}

In fact opportunistic routing is considerably different from normal routing mechanisms. In conventional routing approaches, the next hop is fixed and is responsible for sending data flows and therefore this kind of routing is not suitable for networks like VANET that their topology is dynamically changing and consequently packet drop probability is high. In contrast in opportunistic routing instead of selection of a fixed next hop, under certain circumstances a set of potential forwarders are selected which are able to continue forwarding the packets to the receiver(s) node(s). Maximum coding gain is possible if we select next hop based on highest coding opportunity on the optimal path to the destination. This optimal path should be determined based on opportunistic metric(s).

\section{RELATED WORK}

A number of opportunistic routing mechanisms have been proposed so far, but in this article our main aim is opportunistic routing approaches based on the network coding condition which is called network-coding aware routing. Network coding-aware routing approaches can be categorized into two main groups, namely active and passive. Furthermore, the passive category can be divided into two sub-categories, centralized and distributed.

\section{A. Centralized and Active Network Coding-Aware Mechanisms}

In centralized coding-aware methods, data propagation mechanism is not distributed and most of the control and procedures are under administration of a single or a set of nodes which are in charge of controlling and performing traffic exchange. One of the first methods in coding-aware routing is COPE [4]. This method was proposed by Katti et al. as a packet forwarding approach. Two other most important centralized coding-aware mechanisms are ROCX method proposed by Bin Ni et al. [5] and CARTR proposed by Lee et al. [6]. In active coding-aware methods, every node requires to gather information from neighbor nodes. But unlike other coding-aware types in this kind of coding-aware mechanism, 
nodes make decisions based on real time information that they receive from their neighbor nodes. ACH method proposed by Jiao et al. [7] and CAMP proposed by Han et al. [8] are active coding-aware methods.

\section{B. Distributed Network Coding-Aware Mechanisms}

In this type of mechanisms, decisions are made locally and every individual node is responsible to make proper decisions based on its local information. Usually these types of approaches are more flexible and the decisions made this way are more accurate. In the centralized NC-aware methods, only a single operating unit handles the entire data propagation procedure. NCAR was proposed by Wei et al. [9]. In this protocol in the process of routing discovery, the path is selected based on possible coding opportunities and link delivery ratio. In the discovery phase, it checks whether current data flow can be mixed with the already established data flow. In prior research works, the decoding process performs only in one-hop (node) regardless of the number of nodes that the coded packet has passed. The NJCAR method [10] eliminates this shortcoming. The main aim of this technique is to seek all possible paths between sources and destinations in order to identify several node decoding opportunities. In NJCAR, the relay node gathers global information about all the nodes in order to select the best path. The RCR protocol which is proposed in [11] assumes that different flows have various rates, unlike existing coding protocols which consider that all flows coded together have the same rate. This protocol improves performance by splitting the traffic to escalate coding opportunities. What is more OCAR [12] uses the metric $\mathrm{M}_{\mathrm{CAIA}}$ that take into account so as to consider variations in a link's bandwidth.

The Markovian routing metric protocol developed by $\mathrm{Wu}$ et al. in [13] maintains multiple NC unicast flows. In this protocol the cost of forwarding packets through a link depends on which node a packet arrives from. This protocol uses the Markovian [14] metric for channel resource consumption optimization because of local coding. COAR which was introduced by Yan et al. [15] combines NC and opportunistic routing approaches. It gathers local information of nodes in order to make decisions regarding packet forwarding. ICM [16] is another coding-aware in which the authors introduced a metric keeping in view link interference and coding gain with the aim of achieving a coding-aware and interference-avoidance metric called ICM. Gue et al. in [17] proposed a backbone-based routing that combines backbone routing features with $\mathrm{NC}$ concept. The main aim of backbone routing is to provide a minimum connected spanning tree for a given network.

Singh et al. proposed COREMEN in [18] in which coding decisions for various flows take place on each node separately. Hence synchronization between nodes is not necessary. The main purpose of this method is to balance the forwarding timer in order to eliminate the need for the synchronization between nodes. Hou et al. in [19] proposed CARB, a coding-aware path discovery method that uses time slots to facilitate a balance between link bandwidth and packet forwarding. CARB deals with the issue of maximum available bandwidth calculation for a particular path that supports NC. $\mathrm{NC}$ methods contain several advantages which provide more reliable network architecture, higher throughput and lower packet loss and end-to-end delay. Consequently several techniques have been proposed so far as we mentioned them above. Since there are several shortcomings in discussed methodologies, there is a need for algorithms and methods that mitigate some or all of these drawbacks. So in the remainder we discuss our proposed approach as an extension and improvement for previous methods.

\section{THE PROPOSED APPROACH}

We propose an approach called Opportunistic Network Coding-aware Data Dissemination in Vehicular Ad-hoc Networks (NCODWN). This approach contains several modules with each module carrying certain responsibilities and having a specified role in the data propagation procedure. All these procedures, metrics and modules are executed as a single integrated system in which every component has its own responsibility.

\section{A. Information Gathering}

In the initial stage nodes need to gather sufficient, accurate required information - information that is used in different modules and encoding decision making processes. The data is collected via three different sources, namely overhearing, reception reports and decoded packets. Once a node has retrieved the necessary information from its neighbor nodes, it begins performing predefined algorithms and actions.

\section{B. Reception Report}

A reception report is a kind of message containing local information on each node which is periodically sent at predefined time intervals by nodes to their own neighbors. This report contains a node's coding table and a sort of packet pool containing assorted local information regarding the node. The only instance in which the whole table should be forwarded based on the predefined schedule is when major changes or modifications took place on the majority of the existing information within the coding table.

\section{Encoding}

Encoding is a procedure of mixing (XOR) packets at an intermediate node which is responsible for encoding packets of different flows based on existing information in order to improve throughput. The coding procedure is arranged by using opportunistic coding, a mechanism in which encoding decisions are made dynamically. The process input consists of the information gathered by each node. With such information at hand, the algorithm decides which packets need to be coded together. Our designed coding table includes the Se_Neighb field which represents neighbor nodes from which packets were received. No_Hop represents the number of hops that a packet traverses to get to the node. Tra_Node indicates the identification of each node that packets pass through to reach the node. Re_Node shows the node(s) that need this packet and $\mathrm{Cu}$ _Node is the field node(s) to which the packet is being forwarded. Every node in the network has a table to save its own information as well as data received from neighbors. Later a node will utilize this information to encode different data flows together. Coding table is represented in Fig. 2. 


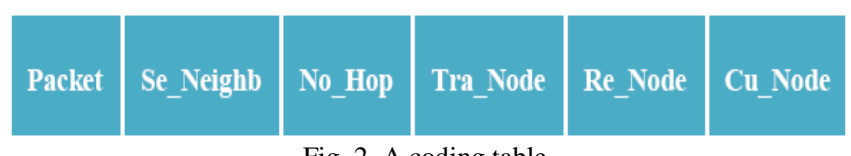

Fig. 2. A coding table.

\section{1) Encoding decision making}

Encoding decision making is a crucial element of the proposed approach as it has a direct relation to coding gain and network throughput. Once the node, based on its current information recognizes that it is in the position of an encoder, it executes the coding algorithm. Encoding must take place in such a way that as it results in the maximum (possible) number of packets to be encoded together. In the coding procedure it must be ensured that receivers can decode the packets in order to retrieve the information. If more than one packet shall be forwarded, the paths need to be chosen and prioritized so as to define a metric called Coding Delivery Ratio (CDR). The path with higher CDR is selected as the best path while the one with the second best CDR will be selected as second best path and so on. If we consider path $P=n_{1}$, $n_{2}, \ldots n_{h}$, the overall CDR for a given path $P$ is:

$$
\mathrm{CDR}_{n}=\frac{\# D e P a}{\# \operatorname{RePa}} \quad \mathrm{CDR}_{p}=\sum_{i=1}^{h}\left(n_{i}\right)
$$

Refer to (1), $\mathrm{DePa}$ represents the number of delivered packets and $\mathrm{RePa}$ stands for the number of packets requested by the node. The path with the highest amount of $\mathrm{CDR}_{p}$ will be designated as the best path. The coding algorithm assesses common packet destinations and whether it is possible for the destination node to decode the encoded packets. Moreover it selects the best path based on the CDR range. Table I highlights the coding process.

TABLE I: CODING PROCESS PSEUDO-CODE

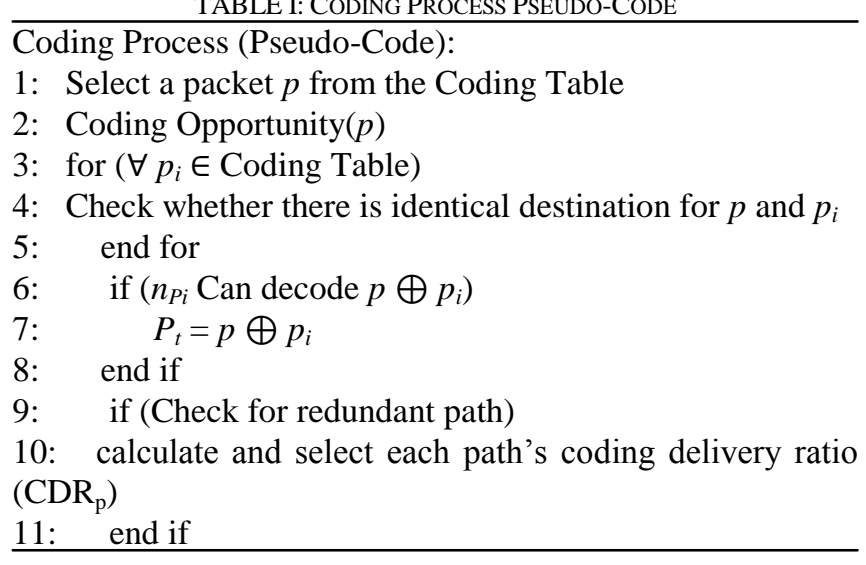

\section{2) Forwarding module}

Upon coding process completion, forwarder nodes ought to be selected. In order to increase reliability and reduce the risks of packet forwarding, and also to address the dilemma of best forwarder selection, we define a priority-based node selection process. In this technique, nodes are sorted according to their coding possibility which can be calculated by a summation of the number of possible packet encoding chances for the node. Based on the CP of every node a timer needs to be defined which indicates at which time packets should be forwarded to the destination as it is indicated in (2).

$$
\text { Timer }=\frac{1}{C P}
$$

A higher CP and lower timer equals to nodes with better coding possibility. Such nodes will thus forward packets earlier. Following the best forwarder selection, nodes must forward the data flow packets. In our approach a multiple-unicast session was applied instead of broadcast with the intention of enhancing reliability and packet loss detection within the data propagation procedure.

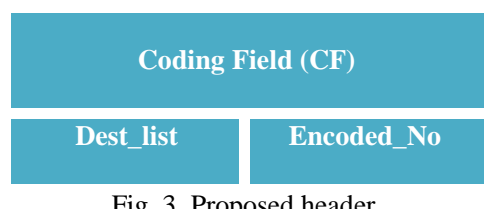

Fig. 3. Proposed header.

However if we wish to use multiple-unicast sessions as opposed to broadcast, some packet modifications are required. Due to the characteristics of unicast sessions, in unicast sessions, messages are supposed to be delivered to only one destination. In the current approach, it may be necessary to send a packet to multiple destinations. So in order to eliminate this limitation, we define a new packet header called Coding Field (CF) as depicted in Fig. 3. Dest_list represents the list of all packet destinations and Encoded_No represents the number of coded packets. CF is capable of saving several destination node addresses. Upon receiving a packet, if node recognizes its address is mentioned on the destination list, it processes the packet.

\section{Decoding}

Once an encoded packet reaches its destination node, the receiver attempts to decode the packet using information in its coding table. The decoding procedure is deemed successful only if the destination has sufficient information to retrieve the packet. Otherwise it cannot retrieve the original packets and the decoding procedure fails. Each encoded packet applies the coding process as depicted in Table II.

In the course of decoding, the node first checks whether the packet is original or encoded. If it is original, it simply adds it to its coding table. Otherwise the node obtains original packets from its packet pool and attempts to XOR them to the received encoded packet and retrieves the original packet.

\section{E. Monitoring}

The monitoring module is responsible for overseeing the overall coding operations. Its principal aim is to verify the network load and end-to-end delay parameters. If either of these parameters does not fall into an acceptable range, the monitoring module cancels or modifies the coding procedure in order to maintain a balance between throughput and end-to-end delay.

Secondly, the main advantage of NC is its excellent functionality in heavy load traffic network conditions with the least acceptable amount of traffic. Therefore if traffic is extremely light, the coding module might be turned off to save resources. 
TABLE II: DECODING PROCESS PSEUDO-CODE

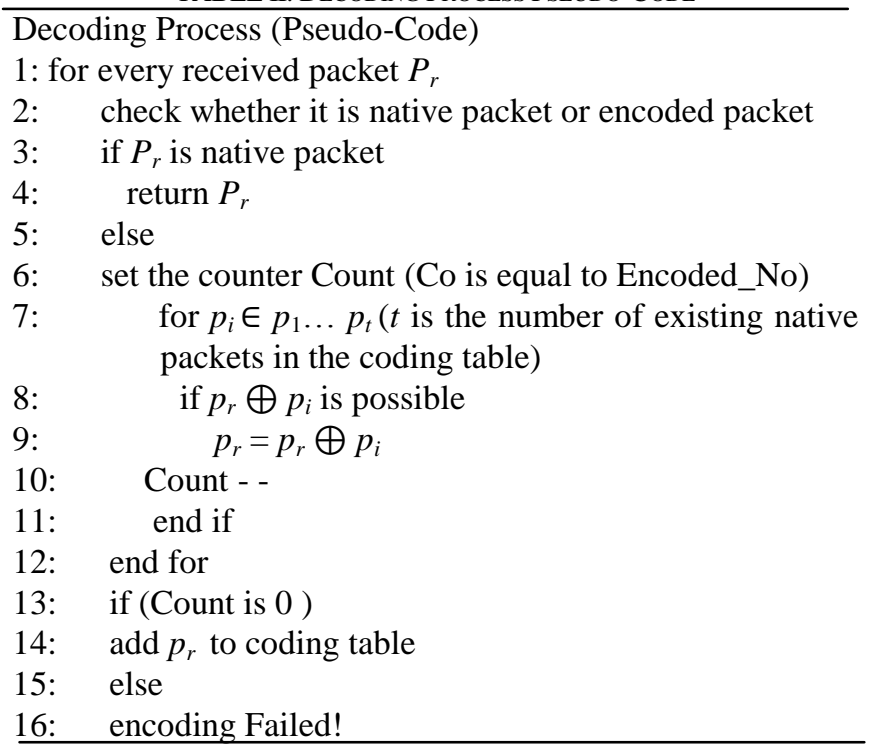

Load metric (LM) is calculated every 60 seconds and its minimum value is 1 . If it drops below this predefined level, then the NC procedure stops as depicted in (3):

$$
\text { Load Metric }(\mathrm{LM})=\frac{\text { Forwarded Packets }}{\text { Number of Nodes }}
$$

The importance of this type of control is that it facilitates integrating the whole system which making it flexible to any sudden change in the network. Moreover it mitigates the possibility of collisions and packet loss within the network.

\section{Simulation Results}

Network throughput determines the total of data that successfully received at the destination node per unit of time. The time unit is usually per second. The higher the throughput the more amounts of data transferred in the same amount of time in the network. It means if we manage to improve the throughput of the network, consequently it helps us to use our resources efficiently. Moreover the higher amount of delivery ratio indicates that packet loss rate is lower and protocol is more efficient from the perspective of data delivery.

A point to note is that late packets received can be useless even with high packet delivery. We ran the simulation based on two methods, COPE and our proposed approach, Opportunistic Network Coding-aware Data Dissemination in Wireless Networks (NCODWN). As COPE is the backbone of coding-aware approaches, most of the discussed approaches in literature have been evaluated compared with COPE. Accordingly we compare the results of our proposed method (NCODWN) with COPE as well. We cached delivery ratio of the both scenarios.

In Fig. 5, we compared this two with each other. In both scenarios, total number of nodes and flows are identical Moreover in order to make sure that in both scenarios every parameter is same, we predefined the mobility structure of the nodes. It means in both scenarios, movement direction of nodes and their speed are identical.

As it is depicted in Fig. 5 at the start of the simulation, between seconds 40 and 70, the amount of packet delivery is high but with the increase of the network load (number of data flows) packet delivery decreases in both approaches. However at the same time, the amount of packet delivery in NCODWN (except for three times in seconds 65, 100 and 236) in most of the times is higher than COPE. It means our proposed method is more robust and the amount of packet lost is less than COPE. Every time that an encoder node encode packets, the related information such as number of packets coded together and number of the transmissions carrying the encoded packets are saved by the encoder node.

Fig. 4 illustrates the percentage of encoded packets into a single transmission. When the number of encoded packets is equal to two, COPE has slightly better performance compared to our approach. However by using NCODWN, the network is able to generate more encoded transmissions compared to COPE when the number of encoded packets is larger than two. Therefore in this case, an encoded transmission carries more information in our proposed approach. Besides the maximum number of packets encoded together are 5 per transmission and none of the methods encoded 6 packets together.

As is depicted in Fig. 6, end-to-end delay for our proposed method is lower than the COPE in most of the times. Moreover in both methods, the flow of fluctuation is almost same as in either of cases all of the parameters of simulation are identical. The main difference is the amount of delay which is higher in COPE.

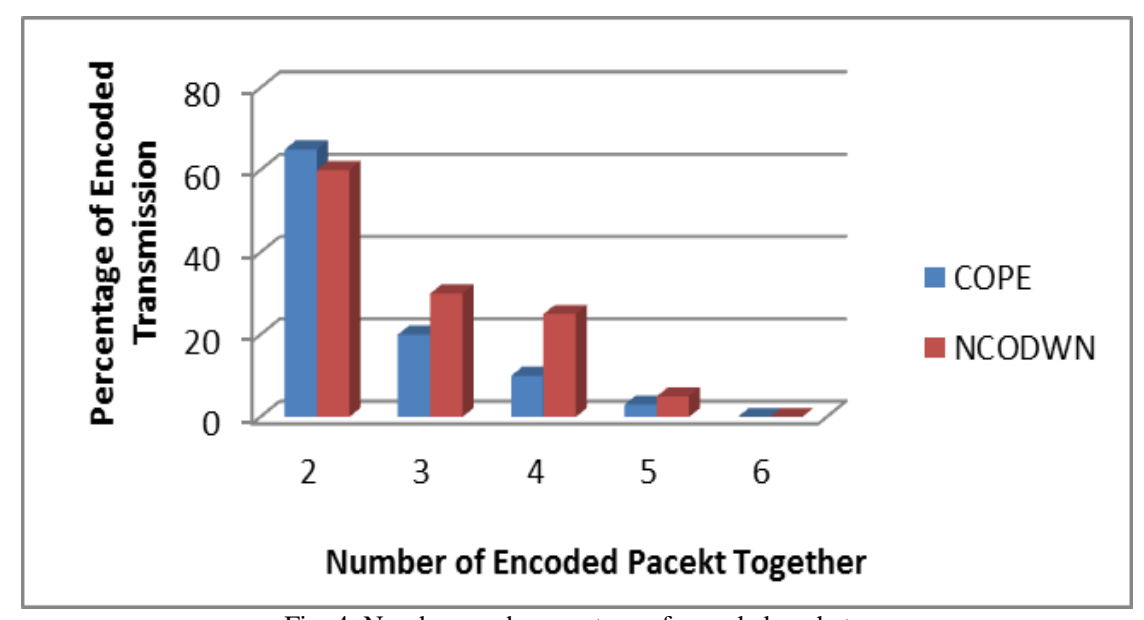

Fig. 4. Numbers and percentage of encoded packets. 


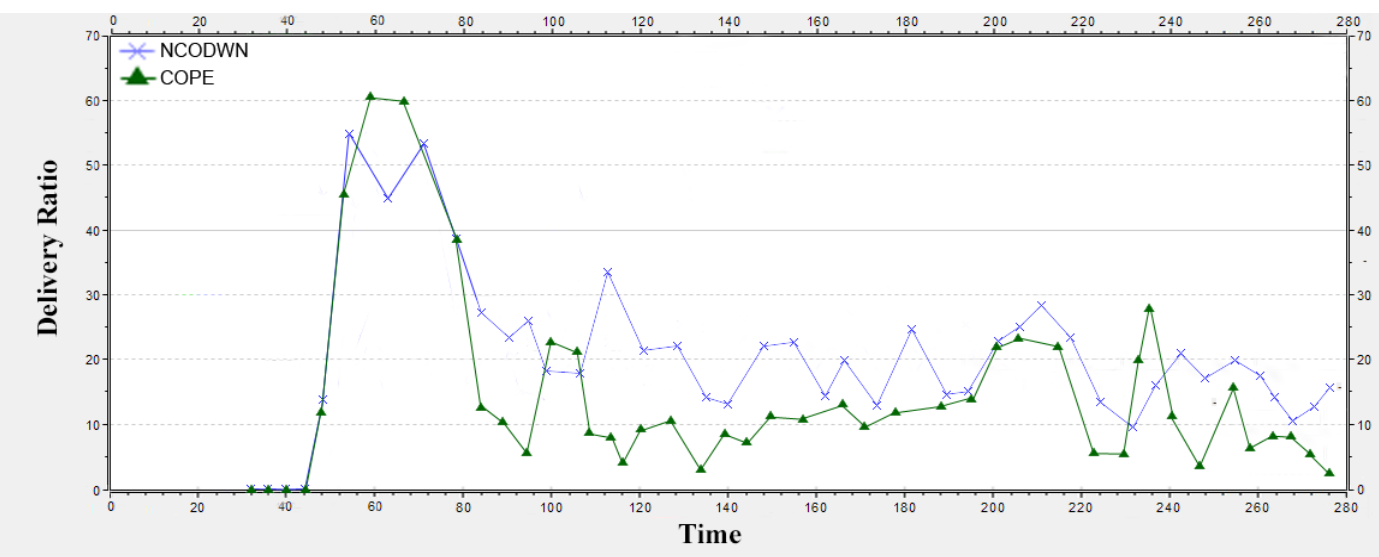

Fig. 5. Delivery ratio comparison between NCODWN and COPE.

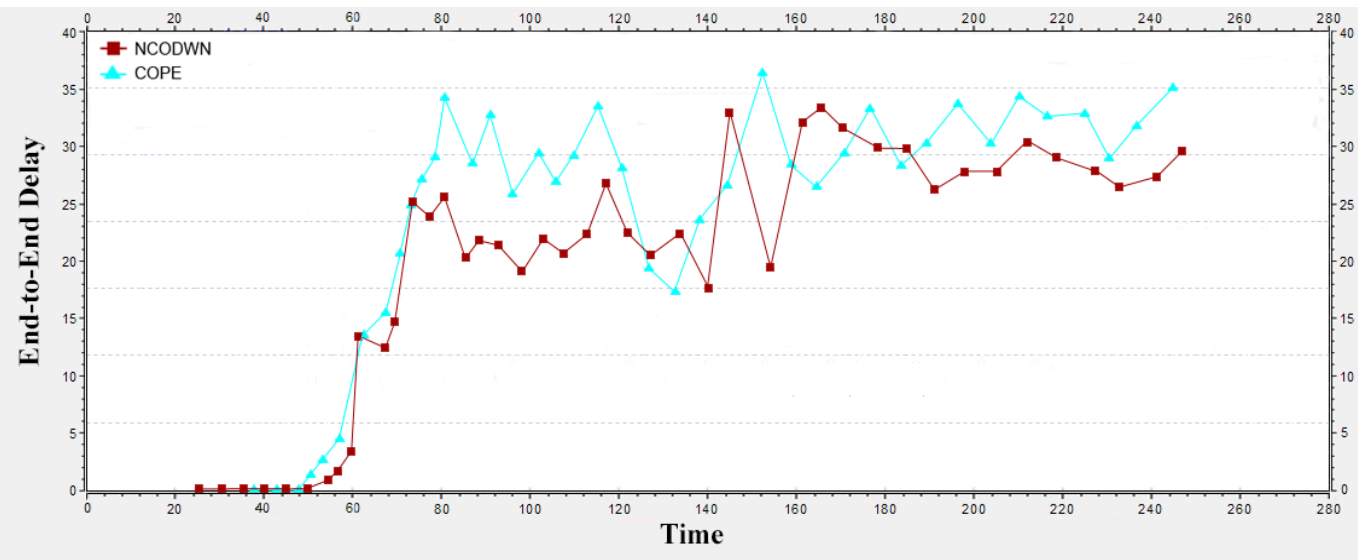

Fig. 6. End-to-end delay comparison between NCODWN and COPE.

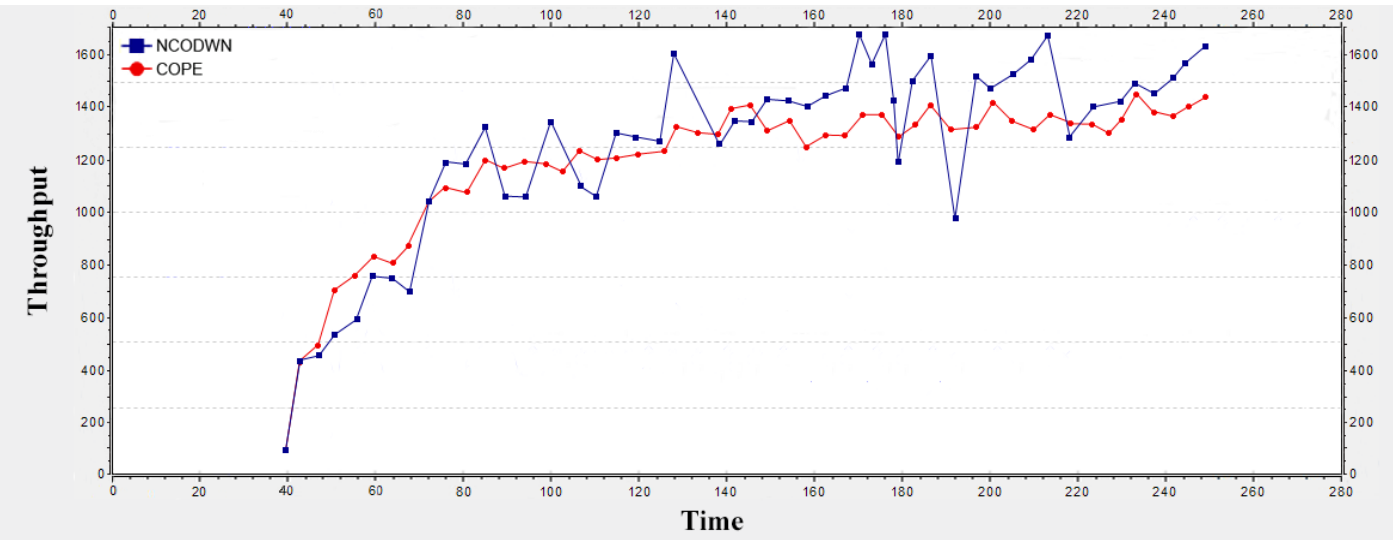

Fig. 7. Throughput comparison between NCODWN and COPE (First Run).

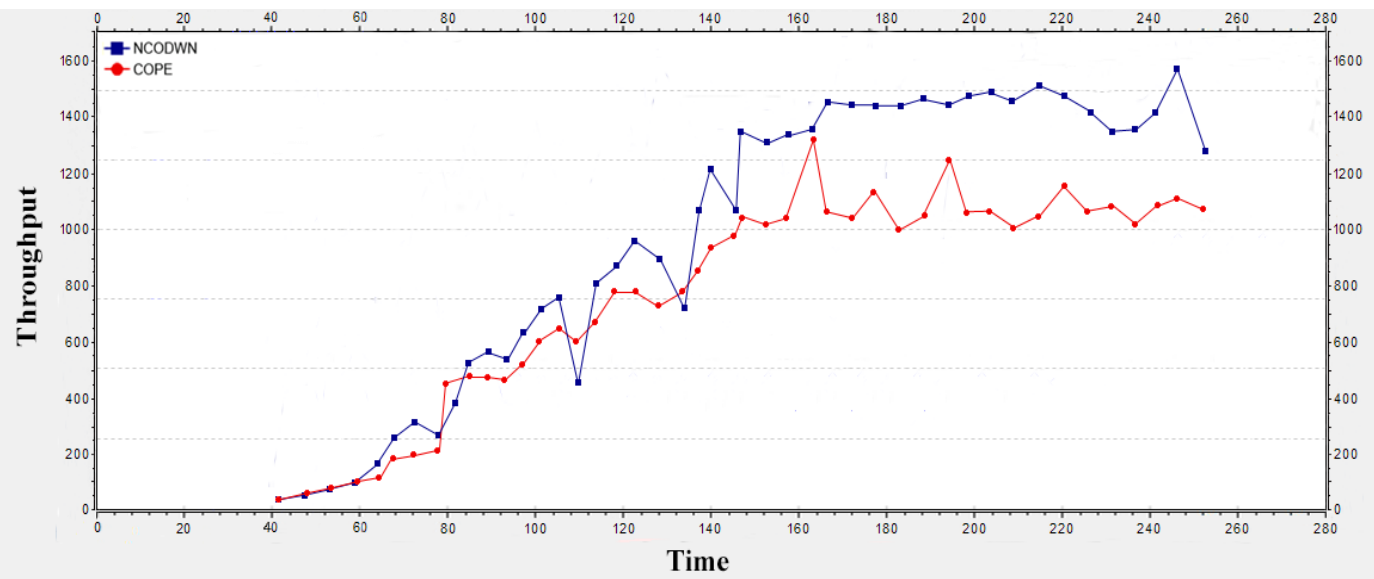

Fig. 8. Throughput comparisons between NCODWN and COPE (Second Run). 
As both throughput comparisons obtained from two simulation runs are depicted in Fig. 7 and Fig. 8, in both COPE and NCODWN methods throughput rates are increasing with passing of time. Average throughput in COPE is steady and in our method is more fluctuating. Although at the same points, NCODWN drops less that the COPE, in most cases network throughput in NCODWN is higher than COPE and in conclusion NCODWN managed to surpass COPE.

\section{CONCLUSION}

In this article, we aimed to improve the existing data propagation method in vehicular ad-hoc networks by implementing and enhancing various modules and metrics involved in data propagation process. Our methodology is composed of several elements such as transmission module, packet pool module, monitoring module and other metrics. Therefore we proposed an approach called Opportunistic Network Coding-aware Data Dissemination in Wireless Networks (NCODWN). Unlike COAR, our approach is functional when nodes are either fixed or mobile and also when topology changes occur. In addition network load is continuously captured and monitored to mitigate packet loss and possible collisions. We employed a differentiate method in the information gathering phase which eliminates unnecessary message forwarding and reduces message size. Also a novel packet pool and a new metric for forwarding prioritization phase is implemented. Simulation results demonstrate that performance of COPE and COAR is improved by our proposed approach (NCODWN) in terms of packet delivery, end-to end delay and average throughput.

\section{ACKNOWLEDGMENT}

This Research is supported by the Ministry of Higher Education (MOHE) and University of Malaya (project no.HIR-MOHEB00009)

\section{REFERENCES}

[1] R. Ahlswede, N. Cai, S. Y. Li, and R. W. Yeung, "Network information flow," IEEE Transactions on Information Theory, vol. 46 pp. 1204-1216, 2000.

[2] D. Aguayo, J. Bicket, S. Biswas, G. Judd, and R. Morris, "Link-level measurements from an 802.11 b mesh network," ACM SIGCOMM Computer Communication Review, vol. 34, pp. 121-132, 2004.

[3] M. A. Iqbal, B. Dai, B. Huang, A. Hassan, and S. Yu, "Survey of network coding-aware routing protocols in wireless networks," Journal of Network and Computer Applications, vol. 34, pp. 1956-1970, 2011.

[4] S. Katti, H. Rahul, W. Hu, D. Katabi, M. Médard, and J. Crowcroft, "XORs in the air: practical wireless network coding," IEEE/ACM Transactions on Networking, vol. 16, pp. 497-510, 2008.

[5] B. Ni, N. Santhapuri, Z. Zhong, and S. Nelakuditi, "Routing with opportunistically coded exchanges in wireless mesh networks," in Proc. 2nd IEEE Workshop on Wireless Mesh Networks, 2006, pp. 157-159.

[6] S. H. Lee, J. Kim, and H. G. Cho, "Coding-aware real-time routing in multi-hop wireless sensor networks," in Proc. 2011 IEEE International Conference on Consumer Electronics, 2011, pp. 443-444.

[7] X. Jiao, X. Wang, and X. Zhou, "Active network coding based high-throughput optimizing routing for wireless ad hoc networks," in Proc. 4th International Conference on Wireless Communications, Networking and Mobile Computing, 2008, pp. 1-5.
[8] S. Han, Z. Zhong, H. Li, G. Chen, E. Chan, and A. K. Mok, "Coding-aware multi-path routing in multi-hop wireless networks," in Proc. IEEE International Conference on Performance, Computing and Communications, 2008, pp. 93-100.

[9] X. Wei, L. Zhao, J. Xi, and Q. Wang, "Network coding aware routing protocol for lossy wireless networks," in Proc. 5th International Conference on Wireless Communications, Networking and Mobile Computing, 2009, pp. 1-4.

[10] Z. Zhou and L. Zhou, "Network joint coding-aware routing for wireless ad hoc networks," in Proc. 2010 IEEE International Conference on Wireless Communications, Networking and Information Security, 2010, pp. 17-21.

[11] Y. Yan, Z. Zhao, B. Zhang, H. T. Mouftah, and J. Ma, "Rate-adaptive coding-aware multiple path routing for wireless mesh networks," in Proc. 2008 IEEE Global Telecommunications Conference, 2008, pp. $1-5$.

[12] J. Z. Sun, Y. A. Liu, H. F. Hu, and D. M. Yuan, "On-demand coding-aware routing in wireless Mesh networks," The Journal of China Universities of Posts and Telecommunications, vol. 17, pp. 80-92, 2010.

[13] Y. Wu, S. M. Das, and R. Chandra, "Routing with a markovian metric to promote local mixing," in Proc. 26th IEEE International Conference on Computer Communications, 2007, pp. 2381-2385.

[14] A. Konrad, B. Y. Zhao, A. D. Joseph, and R. Ludwig, "A Markov-based channel model algorithm for wireless networks," Wireless Networks, vol. 9, issue 3, pp. 189-199, May 2003.

[15] Y. Yan, B. Zhang, H. T. Mouftah, and J. Ma, "Practical coding-aware mechanism for opportunistic routing in wireless mesh networks," in Proc. IEEE International Conference on Communications, 2008, pp. 2871-2876.

[16] Y. Lu, C. Shen, Q. Xia, and J. Tao, Wireless Routing, No. 60603067 , pp. 1-4, 2009.

[17] H. Guo, Y. Qian, K. Lu, and N. Moayeri, "Backbone routing over multihop wireless networks: increased network coding opportunity," in Proc. 2010 IEEE International Conference on Communications, 2010, pp. 1-5.

[18] J. Islam and P. K. Singh, "CORMEN: coding-aware opportunistic routing in wireless mess network," Journal of Computing, vol. 2, no. 6 , June 2010.

[19] R. Hou, K. S. Lui, and J. Li, "Coding aware routing in wireless networks with bandwidth guarantees," in Proc. 2011 IEEE 73rd Vehicular Technology Conference (VTC Spring), 2011, pp. 1-5.

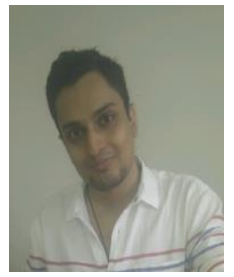

Hossein Malakooti received his master degree of data communication and networking at the University of Malaya, Faculty of Computer Science and Information Technology. He had finished his bachelor's degree in information technology engineering in Azad University, South Tehran Branch in 2010, he decided to join the research community at the University of Malaya.

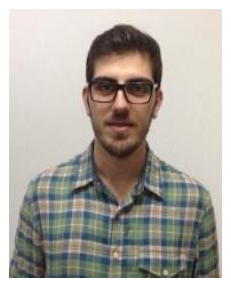

Ali Jalooli received his bachelor's degree in computer science from the Staffordshire University in 2012. He is currently pursuing his master's degree in computer science and has been a research assistant since May 2013 at the University of Malaya, KL, Malaysia. His research interests include vehicular ad hoc network, quality of service, vehicle traffic routing and applications of intelligent transportation system.

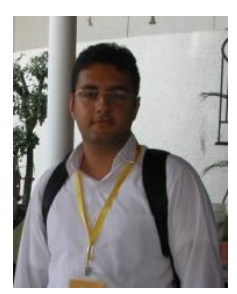

Mohammad Reza Jabbarpour is currently a $\mathrm{PhD}$ candidate and research assistant in the System and Technology Department, Faculty of Computer Science and Information Technology at University of Malaya, Malaysia. He received his master degree of data communication and networking in 2012 at the same university. His current research interests include ad hoc networks, quality of service, swarm intelligence and vehicle traffic routing systems. 


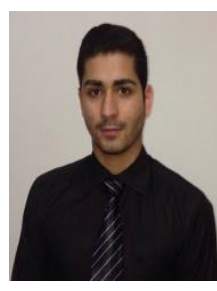

Alireza Marefat Vayghani is now pursuing his master's degree in computer science at University of Malaya, Malaysia, Kuala Lumpur and in 2012 he gained his bachelor's degree in information technology with specialism in networking from Staffordshire University. His current interests comprise vehicular ad hoc network and road safety risk reduction.

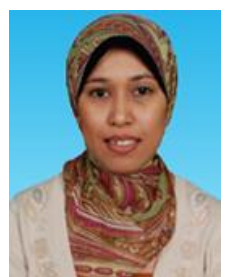

Rafidah Md Noor received her BIT from Universiti Utara Malaysia in 1998, and MSc. degree in computer science from Universiti Teknologi Malaysia in 2000 , and $\mathrm{PhD}$ in computing from Lancaster University in 2010. She is currently a senior lecturer at Computer System and Technology Department at the Faculty of Computer Science and Information Technology, University of Malaya. Her research interests include network mobility, vehicular networks, mobile IP, quality of service and quality of experience. 